\title{
Cities and Cellular Automata
}

\author{
ROGER WHITE \\ Department of Geography, Memorial University of Newfoundland, St. John's, Newfoundland, A1B 3X9, Canada
}

(Received 7 December 1997)

\begin{abstract}
Cellular automata provide a high-resolution representation of urban spatial dynamics. Consequently they give the most realistic predictions of urban structural evolution, and in particular they are able to replicate the various fractal dimensionalities of actual cities. However, since these models do not readily incorporate certain phenomena like density measures and long-distance (as opposed to neighbourhood) spatial interactions, their performance may be enhanced by integrating them with other types of urban models. Cellular automata based models promise deeper theoretical insights into the nature of cities as self-organizing structures.
\end{abstract}

Keywords: Cellular automata, Urban spatial dynamics, Model integration, Fractal dimensions, Complexity

\section{INTRODUCTION}

As computing power continues to expand, microsimulation models become increasingly interesting as ways to understand complex systems. The approach has much to recommend it, since it gets to the level at which objects or agents act to structure a system, and thus allows us to explore the phenomenon realistically while imposing a minimum of arbitrary simplifying assumptions. Nevertheless, the strength of the approach is also its weakness: as the scale of the map approaches $1: 1$, it no longer provides an overview of the country. In other words, the detailed nature of explanation inherent in micro-simulation models may make it difficult to understand the general nature of emergent properties. On the other hand, macro-scale models may give extremely useful descriptions of emergent behaviour, even if they do not show how these macroscopic structures arise. Clearly it is desirable to have both levels of description, since each complements the other, and the micro-level provides a deeper understanding of the reasons for the macro-scale phenomena. But even better is to have an approach that has properties of both levels, and provides a link between the two. Cellular automata (CA) are such an approach.

Cellular automata are well suited to urban modelling, being inherently dynamic and intrinsically spatial, with good spatial resolution. Although they are remarkably simple mechanisms they can generate spatio-temporal patterns of unlimited complexity (Wolfram, 1984; Langton, 1990; 1992; Bak Chen and Creutz, 1989), and that is one reason why they are such powerful modelling tools. Nevertheless, cities are even more complex, being 
generated by a large number of processes operating at a variety of spatial and temporal scales. Thus in order to use CA for modelling cities with a useful degree of realism, it is necessary to use more complex CA - that is, CA with more states, a larger neighbourhood, and more complex transition rules. It is also necessary to constrain the CA in various ways in order to represent the effect of processes operating at scales that cannot be directly included in the CA transition rules - by in effect modifying the $\mathrm{CA}$ transition rules as the $\mathrm{CA}$ runs.

\section{DEFINING CELLULAR AUTOMATA FOR URBAN MODELLING}

A CA may be defined as (1) a discrete cell space, together with (2) a set of possible cell states and (3) $a$ set of transition rules that determine the state of each cell as a function of the states of all cells within (4) a defined cell-space neighbourhood of the cell; (5) time is discrete and all cell states are updated simultaneously at each iteration. While the concept is remarkably simple, and many of the most intensively studied CA, like Game of Life are, in fact, almost trivially simple as mechanisms, the definition leaves quite a bit of room for variety, and the CA that have been most useful in urban modelling tend to depart significantly from the ideal of simplicity. It is useful, therefore, to elaborate on this definition from the point of view of urban modelling.

(1) Cell Space. The most important characteristics of cell space are dimensionality, cell size and cell shape, and homogeneity or heterogeneity. CA developed for urban modelling are, not surprisingly, typically defined on a two dimensional cell space, though it is easy to imagine using a three dimensional cell space in order to be able to represent building height, or, more generally, density of land use. One dimensional CA, while less common in modelling applications, are being used for urban traffic modelling (Nagel et al., 1997; Di Gregorio et al., 1996). Cell size in current applications ranges from $250 \mathrm{~m}$ (e.g. White et al., 1997) down to tens of metres (Batty and Xie,
1994). At the latter scale, cells may represent cadastral units (i.e. actual land lots), which may have any shape; thus while it is often convenient for various reasons (e.g. run time) to use a regular cell space, that is not necessary. In conventional CA the cell space on which the cell state dynamics is defined is homogeneous - that is, all cells are identical except for their state. In modelling cities, however, where it is natural to let cell states represent land use or land cover, it is useful to work on a heterogeneous cell space, where each cell may have an intrinsic quality (or a vector of qualities) representing such characteristics of the real geographical space as soil quality, slope, or land use regulations.

(2) Cell State. In most urban modelling applications, cell states represent land use and land cover, but depending on the focus of the model, they may represent other features of the urban area; Portugali and Benenson (1995; 1996), for example, use cell states to represent social categories, e.g. immigrant vs. native populations. And, broadening the idea of $\mathrm{CA}$, the conventional Boolean cell state could acquire a cardinal measure in order to represent such characteristics as building quality or density. Finally, it is often convenient to define some cell states as fixed, so that while cells in these states can influence the state transitions of other cells, they are not themselves subject to changes of state (e.g. White et al., 1997). Rivers and parks are examples of land uses that could well be represented by fixed states.

(3) Transition Rules. These are the heart of CA since they represent the process that is being modelled; they are thus the key to a good application. They may be deterministic or stochastic; very simple (e.g. a cell changes to the modal state of its neighbourhood), or quite elaborate, like the transition rules of the urban model described below in Section 4. Typically the rules are fixed, but some work has been done with simple CA that evolve to solve a test problem, and there is potential for use of $\mathrm{CA}$ with rules that evolve endogenously to represent such phenomena as urban real estate speculation. 
(4) Neighbourhood. Conventionally, the neighbourhood to which the transition rules are applied is small. In two dimensional rectilinear CA the most commonly used neighbourhoods are the Von Neumann (4-cell) and the Moore (8-cell). These are convenient, and for many physical processes, where field effects are absent and interaction occurs only through contiguity, they are entirely appropriate. They are occasionally used for urban modelling (e.g. Cecchini and Viola, 1990; 1992); but in general larger neighbourhoods would seem to be more appropriate, since land use decisions are made by people who are aware of and able to take into account conditions over a larger area than that represented by a Moore neighbourhood. In general, the size of the neighbourhood should be defined with reference to the distance over which the processes represented by the transition rules operate.

(5) Time. Discrete time with simultaneous cell state transitions is standard. However, there may be situations where it is convenient to nest time scales, with some parts of the CA running a number of time steps for each step in the rest of the model. For example, in a model of a region in which some areas are subject to seasonal inundation, the low-lying areas are modelled on a monthly basis to capture the dynamics of the flooding, while upland areas are modelled with a time step of one year (Uljee et al., 1996). A rather different approach to time would be to update cell states sequentially; such an approach would depend on having a method for choosing the sequence of cells that was inherently meaningful in terms of the modelling problem.

\section{CELLULAR AUTOMATA BASED URBAN MODELS}

A number of CA models of urban systems have been developed, together with several modelling systems. Tobler (1979) was the first to propose a cellular approach to geographical modelling, and his idea was followed up by Couclelis $(1985 ; 1988$;
1989; 1996) and later Takeyama (1996). Couclelis uses the ideas of CA modelling to explore the nature of space and spatial relations in the context of dynamics, but does not attempt applications to specific cities. However, she points out that while standard spatial interaction based approaches to the modelling of urban and regional systems postulate a relational space, usually of low resolution, in which absolute location is virtually irrelevant, GIS (Geographical Information Systems), on the other hand, presuppose an absolute space, and one of very high resolution. Both approaches are appropriate for certain problems, but an adequate representation of a city will involve both relational and absolute spaces. As she says,

On the one hand is absolute space and the concrete geo-referenced location or object with attributes, that knows nothing of its surroundings; on the other is relative space and the varified complex spatial relation that is so innocent of the specifics of place as to be dubbed "the geography of nowhere". In between is proximal space and the generalized CA models, that can now ... partake of the earthy data richness of GIS while probing hypotheses about the large scale effects of micro-scale interactions. That these models can now ... be extended into the realm of relative space, where the most robust theories of urban and regional growth are still to be found, is a[n]... exciting prospect. (Couclelis, 1996, p. 174)

Couclelis (1996) and Takeyama (1996) thus propose a generalized modelling language which would permit integrated dynamic spatial modelling at all scales within a GIS framework.

Batty and Xie (1996) and Xie (1996) have developed a CA for urban modelling which generates both land use patterns and an associated transportation network. Their use of a CA to generate a network is interesting and novel, but not yet entirely successful; one problem may be that CA processes are inherently local, whereas the evolution of a transportation network must reflect both local and long range interactions. Their models are not developed to model actual cities, nor do they carry out sensitivity analysis to determine general 
characteristics of model behaviour. They conclude that

It is unlikely that current approaches can really generate acceptable levels of performance in terms of the goodness-of-fit to existing patterns in the same way in which less complex, traditional models are able, and thus the emphasis in application should not be on fit but on feasibility and plausibility. (Batty and Xie, 1996, p. 203)

It is significant that, like Couclelis, they discuss their CA models in the context of "traditional" (i.e. "Lowry-type") spatial interaction based models, thus recognizing a complementarity. The issue of "goodness-of-fit" is two pronged. On the one hand, CA, by virtue of their spatial detail, permit extremely realistic results; the traditional approaches capture only highly aggregated measures of urban structure, though they may be "realistic" in the sense that an appropriately specified and wellcalibrated model may yield a very good value for a measure of goodness-of-fit. However, this merely brings into stark relief the fact that measures of goodness-of-fit are highly generalized measures, whereas a city is an extremely complex entity. A model capable of producing truly realistic results will thus produce a complex output, and at present there are no appropriate measures of goodness-of-fit. As modelling techniques advance, and with them the quality of the model output, this problem is becoming critical; we will return to it below.

Portugali and Benenson (1995; 1997) and Portugali et al. (1994), while investigating general principles of urban self-organization by means of CA models, have gone farther in the search for empirical realism. They are concerned primarily with urban social structure, and the attitudes which engender and are in turn modified by that structure. They work with models which they characterize as models of human agents in cell space. The cell space represents individual residential lots, and cell states are actually vectors representing such qualities as status and value of the dwelling as well as the characteristics of the family inhabiting it. Transition rules relate changes in dwelling characteristics to the characteristics of families in the cell neighbourhood, and also relate changes in family characteristics (e.g. ethnicity or attitude toward neighbours) to the characteristics of families and dwellings in the neighbourhood. These models clearly demonstrate the close link between basic theoretical ideas and empirical realism that is one of the useful characteristics of cellular automata modelling.

Other applications of CA to urban structure (Cecchini, 1996; Cecchini and Viola 1990; 1992) have emphasized simple automata, for example two state (urban, rural) or small neighbourhood (Von Neumann or Moore) formulations. Makse et al. (1995) have adapted a physical model, a model of correlated percolation in the presence of a density gradient, to the problem of urban form. They use the two state model to simulate the growth of an urbanized area, and show that the result has a fractal structure. The fractal dimensions they generate are almost identical to those characterizing the output of other cellular models (see Section 4 below) (White and Engelen, 1993b; White et al., 1997). Since the latter models do not impose a density gradient, it would seem that that feature of the correlated percolation model is not necessary: a global density gradient can be generated by the local dynamics of the CA itself, a not uncommon phenomenon.

\section{CONSTRAINED CA-BASED URBAN LAND USE MODELS}

White and Engelen (1993a,b; 1994; 1997b), White et al. (1997), and Engelen et al. (1997a) have also developed CA based urban models that join theoretical concerns with empirical realism. These are the only CA based urban models that have been subjected to extensive sensitivity analysis, and also to a degree of empirical testing. It is thus useful to focus on these models both in order to get an indication of the quality of the results possible with CA based urban models, and also to examine more closely 
some of the major issues that arise in urban modelling with CA.

Unlike the models of Portugali and Benenson, which focus on socio-economic phenomena, or those of Batty and Xie which use a cellular mechanism to generate a transport network, these are essentially land use models. The focus is specifically on the dynamics of land use and land cover, while the transport network is taken as given (though it may be altered or extended exogenously during a simulation). Land use and land cover types are divided into two categories: fixed features, like water bodies and parks, and active land use functions like housing, commerce, and industry. In the neighbourhood dynamics defined for the CA, fixed features appear as arguments in the transition rule functions, and so affect the change of state from one active land use function to another, but they are not themselves subject to state changes (other than changes determined exogenously to the CA mechanism itself). Like the fixed features, the transportation network may affect the transition probabilities of active cells, but is not itself changed by the cellular dynamics.

The transition rules applied to active cells incorporate five types of factors:

- intrinsic suitabilities representing inhomogeneities in the geographic space being modelled (e.g. soil quality or legal restrictions);

- the neighbourhood effect, representing the attractive or repulsive effects of the various land uses (cell states) in the neighbourhood consisting of the 113 cells lying within a radius of 6 cells of the target cell;

- a local accessibility effect, representing ease of access to the transport network;

- a stochastic perturbation capturing the effect of imperfect knowledge and varying needs and tastes among the implicit actors whose decisions are represented by cell state transitions;

- global constraints on the number of cells in each state, reflecting the fact that the requirements for land for various activities are largely determined exogenously to the internal land use dynamics of the city being modelled, depending rather on such factors as the size of the city and the need for a balance among various activities. Thus at each iteration, a target number of cells for each state is specified, and the CA determines not the number of cells in each state, but only their location. The target numbers for each cell state may simply be read from a file, or generated by a linked model as described below.

The first four factors are incorporated into the calculation of a vector of transition potentials for each active cell - that is, a potential is calculated for each possible (active) cell state. Then the transition rule is applied: each cell is changed to the state for which it has the highest potential, subject to the global constraints.

The potentials are calculated as follows:

$$
P_{h j}=v a_{j} s_{j}\left(1+\sum_{k} \sum_{i} \sum_{d} m_{k d} I_{i d}\right)+H_{j}
$$

where:

$P_{h j}$ is the transition potential from state $h$ to state $j$;

$m_{k d}=$ a weighting parameter applied to cells with state $k$ in distance zone $d$ (cells that are closer usually are weighted more heavily; but weights may be positive, representing an attractive effect, or negative, if two states are incompatible);

$I_{i d}=1$ if the state of cell $i=k$, otherwise $I_{i d}=0$, where $i$ is the index of cells within the distance zone $d$ (the role of $I_{i d}$ is simply to ensure that only the relevant weight $m_{k d}$ for the cell at location $i, d$ - i.e. the weight corresponding to cell's actual state - is included in the calculation);

$H_{j}=$ an inertia parameter, with $H_{j}>0$ if $j=h$; otherwise, $H_{j}=0\left(H_{j}\right.$ increases the likelihood that a cell will remain in its current state);

$s_{j}=$ the suitability of the cell state for $j$, where

$0 \leq s_{j} \leq 1$;

$a_{j}=$ the accessibility parameter,

$$
a_{j}=\left(1+D / \delta_{j}\right)^{-1}
$$

where $D$ is the Euclidean distance from the cell to 
the nearest link of the network and $\delta_{j}$ is a coefficient expressing the importance of accessibility on the desirability of the cell for land use activity $j$. Accessibility is thus defined strictly locally, in terms of distance to the network, rather than globally in terms of distances through the network to distant points; $v=$ the stochastic disturbance term,

$$
v=1+(-\ln (r))^{\alpha}
$$

where $(0<r<1)$ is a uniform random variate and $\alpha$ is a parameter that allows the size of the perturbation to be adjusted.

The random term permits the cellular city to explore its state space, since cells that are apparently unsuited to a particular activity will nevertheless occasionally be converted to that state. Since the inertia term will normally prevent a cell from reverting immediately to its previous state, these cells may function as nuclei of new developments by increasing the transition potentials of other cells in their neighbourhood.

This generic cellular model has been used to generate test cities (Fig. 1) on both a homogeneous cell space (i.e. space with no suitabilities or transportation network) and a cell space homogeneous except for the presence of a transportation network, for purposes of sensitivity analysis. It has also been used as the basis for simulations of actual cities and regions (Figs. 2, 4). The results are surprisingly realistic for such a simple model, especially for one lacking any representation of long-distance interactions.

The most striking feature is that all cellular cities generated with versions of this generic model exhibit a fractal form:

- The perimeter of the urbanized area (as defined by cells in any state representing an urban land

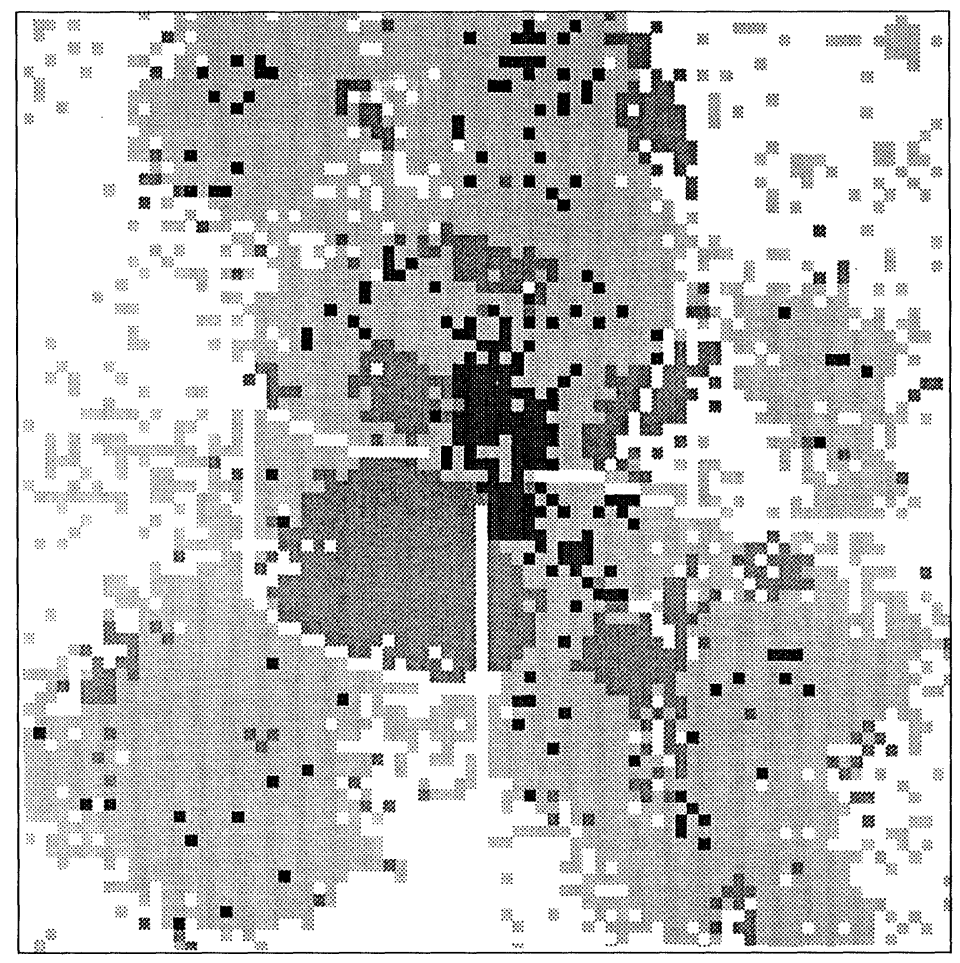

FIGURE 1 Simulation of a generic city generated on a homogeneous cell space, with a transport system represented by "road" cells. Land uses: dark, commerce; medium, industry; light, housing. 
use like housing or commerce) is a fractal (White and Engelen, 1993b).

- The radial dimensions, calculated as the derivative of $\log ($ area $)$ with respect to $\log$ (radius), of both the urbanized area and of individual urban land uses are bi-fractals. For example, the urbanized area is characterized by a radial dimension of approximately 1.92 out to a critical radius; beyond that radius the dimension falls to a value in the neighbourhood of 1.1. Inside the critical radius the cellular pattern is relatively stable, representing an urbanization process that is essentially complete; outside it, the cell state configurations change rapidly, as the CA generates the future urban form (White and Engelen, 1993b; White et al., 1997).

- The radial dimensions of the individual land uses are significantly different from each other, reflecting a relative concentration of the various land uses in successive concentric zones, with, for example, commerce relatively concentrated toward the centre and housing toward the periphery (White and Engelen, 1993b; White et al., 1997).

- The size-frequency spectra of land use clusters are log-linear, another measure of fractal structure (White and Engelen 1993b; White et al., 1997).

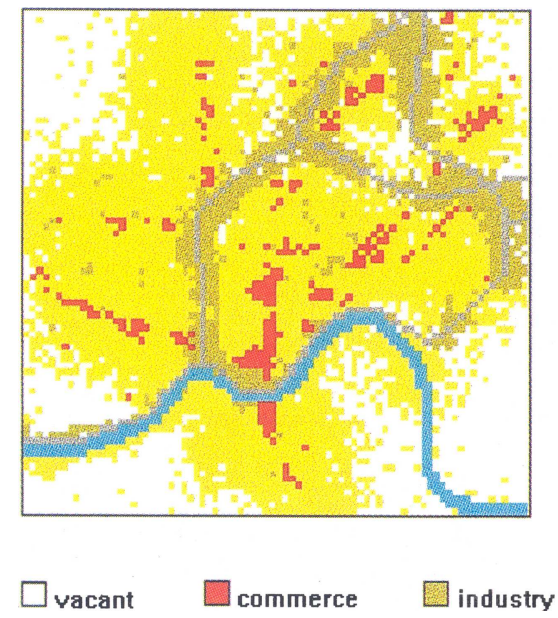

In all cases, actual cities are found to exhibit the same fractal dimensionality (White and Engelen, 1993b; White et al., 1997; Frankhauser, 1991; 1994). Frankhauser (1994), for example, found that for 17 of 19 world cities, the radial dimension of the urbanized area within the critical radius ("rayon de ségrégation") ranged in value between 1.94 and 1.99. 1960 Land use data for Cincinnati, USA, yielded a log-linear size-frequency spectrum with a slope of 1.29 for clusters of commercial land; a cellular simulation of the city to the same year also gave a log-linear spectrum, with a slope of 1.44 (White et al., 1997). And Batty and Longley (1994) determined the fractal dimension of the perimeter of the Cardiff, UK, urbanized area to lie in the range of 1.2-1.3. Thus in terms of their various fractal dimensions, cellular cities would appear to be highly similar to actual cities.

Fractal dimensions are, however, highly general measures. One of the potential strengths of cellular modelling lies in the spatial detail, and hence the realism, that it can provide; and while fractility is a measure of the complexity of the urban form, it is a very abstract one, and thus does not capture the particular form of specific cities or their cellular simulations. Cellular simulations of actual cities, for example Cincinnati (Fig. 2), using the actual transportation network of major roads and

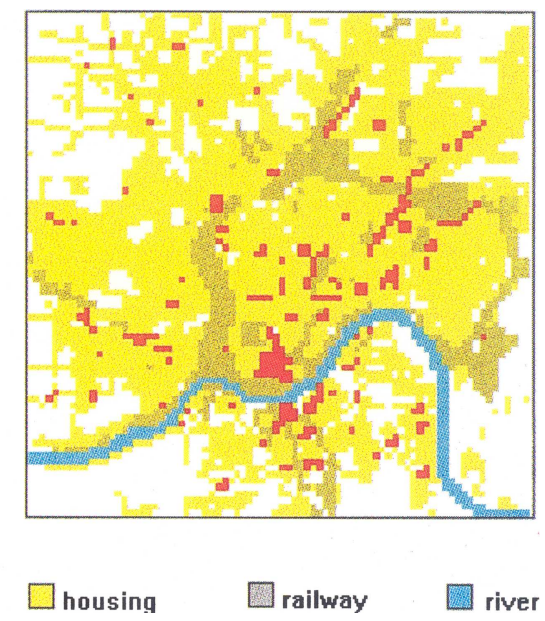

FIGURE 2 Simulation of Cincinnati (left $)$ and actual land use (simplified), 1960 (right); the road system used in the simulation is not shown. See Colour Plate I. 
suitability maps representing the slope of the land, give results that are visually quite similar in form to the land use map of the actual city.

In the case of Cincinnati, the correspondence of the simulated map to the actual map of the city is confirmed by Kappa index values (see Monserud and Leemans, 1992) ranging from 0.51 (fair) for commerce to $0.63-0.69$ (good) for the other land uses. However, Kappa is based on a cell-by-cell coincidence matrix, and so frequently does not capture the more general similarity of patterns. This problem is evident in the comparison of the simulated and actual locations of commercial activity (Fig. 3), where it is clear that the two locational patterns are quite similar, even though very few of the cells coincide. A recently developed map comparison technique making use of fuzzy set theory (Power, 1998) is based on a comparison of local configurations as opposed to individual cell coincidences. An application to the Cincinnati case gives similarity values about $15 \%$ higher than the Kappa index. Thus while the various quantitative measures of the modelling results support the

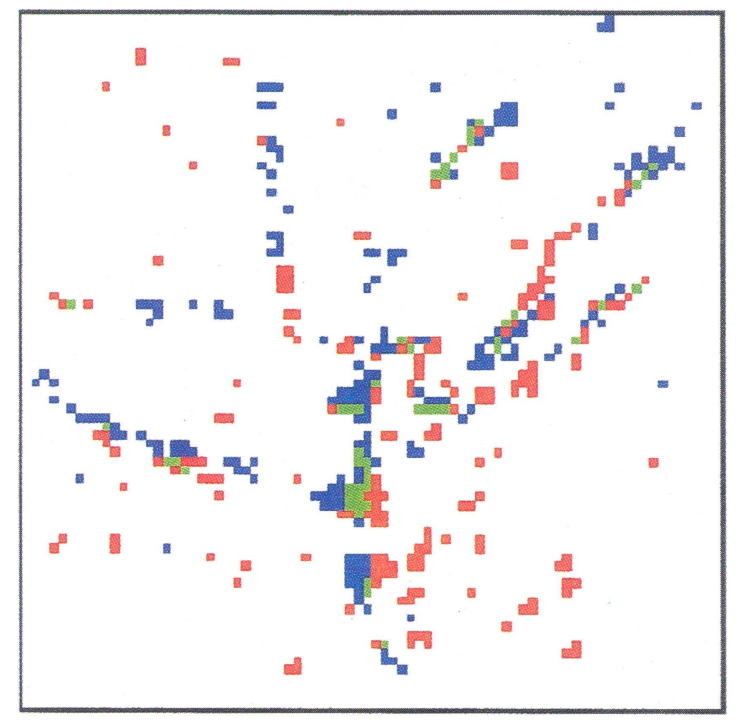

In map 1, $\square$ In map 1, $\square$ In map 2, not in $2 \quad$ in map $2 \quad$ not in 1

FIGURE 3 Comparison of simulated and actual locations of commerce, for simulation shown in Fig. 2. See Colour Plate II. conclusion that the cellular approach is worthwhile, they serve primarily to reveal the inadequacy of the current kit of techniques for assessing spatial patterns - a problem noted by Mandelbrot (1983) among others. In other words, our ability to model reality is outrunning our ability to evaluate the results.

\section{LIMITATIONS OF CA DUE TO SHORT RANGE INTERACTIONS}

While it is gratifying that the results of modelling with cellular automata are as realistic as they appear to be, it is also, in one respect, somewhat puzzling. The fractal nature of the patterns generated by calibrated CA models implies spatial structure at all scales - i.e. spatial autocorrelation up to the dimension of the grid (e.g. $80 \times 80$ cells) - even though the processes that generate the structure operate only over short distances (e.g. 6 cell units). There is no question about how this happens: the iterative CA process can, given sufficient time, transmit a signal from any cell on the grid to any other. Rather, the mystery is why the results are so realistic, since in real cities the spatial interactions that generate spatial structure occur not only at a local scale corresponding to the CA neighbourhood, but also at regional or long distance scales which are not represented at all in the CA models. Must we to conclude, counter intuitively, that the enormous volume of long distance interactions within cities - shopping trips, for example, or the daily trips to work - are irrelevant to the spatial structuring of the city?

Most existing models of the dynamics of urban spatial structure (e.g. Allen, 1983; 1997; White, 1977; 1978; Wilson, 1974; 1976) are based on representations of these long distance flows; and as we have seen, Couclelis (1996), Takeyama (1996), and Batty and Xie (1996) have reaffirmed the need for models of this sort, as have White and Engelen (1994). Fortunately for tradition and intuition, there are indications that the cellular models do not handle all aspects of urban spatial structure as well 
as the spatial interaction based models. For example, cellular models of the sort characterized by Eqs. (1)-(3) represent land use qualitatively, in terms of land use type, and do so with excellent spatial resolution; but they do not represent quantities such as density. In other words, lacking a measure of intensity, the cellular model, in spite of its spatial resolution, cannot give a good quantitative representation of the location of activity. Thus, if only for such mundane reasons, it will frequently be desirable to supplement a CA model with a traditional spatial interaction based representation of urban structural dynamics. But in addition, there is some evidence that intuition is correct, and that long range interactions are important in shaping the urban spatial structure; this is seen most clearly in the commercial sector.

Major retail centres compete for business at the scale of the entire urban area. The process of competition for customers, which determines the revenues of the centres and thus ultimately their success or failure, must therefore be modelled in a way that captures the long distance spatial interaction involved, for example by means of a Lowrytype model, as mentioned by Batty and Xie. The models of urban retail centre dynamics due to Wilson $(1974 ; 1976)$ and White $(1977 ; 1978)$ are both based on long distance spatial interaction models. Extensive simulation experiments with a similar model (White and Engelen, 1997b) indicate that the system of major retail centres is characterized by two qualitatively different types of stable states, depending on the values of relevant parameters: in one of these a single centre is dominant; in the other (convex) state, a number of centres of similar size dominate the hierarchy. If the system is caused to pass through this bifurcation, it may spend a significant time in a meta-stable phase transition state in which the distribution of centre sizes is log linear, representing a fractal structure (Nicolis, 1989). Between the late 1950s and the early 1980s, most US cities passed through this bifurcation, spending a decade or two in the log linear, fractal transition, and then ending up clearly in the convex state (White and Engelen, 1997b). By contrast, in all cases where the CA model based on Eqs. (1)-(3) was realistically calibrated, commerce was fractally distributed - indeed, with this model it is difficult to generate distributions that match either of the stable configurations. The good agreement between the simulated and actual size distributions of commerce clusters for Cincinnati would thus seem to be a fluke, due to the fact that the simulation was tested against 1960 data, when the city was coincidentally in the transition between steady states.

\section{INTEGRATING CELLULAR AUTOMATA WITH OTHER MODELS}

In view of these problems, and in recognition of the fact that activity patterns in cities are dominated by interactions beyond the scale of a cellular neighbourhood, it would seem obvious to link CA models of urban structure with other, standard dynamical models (Uljee et al., 1996; White and Engelen, 1997b). In this approach the dynamical model operates at a macro-scale, in contrast with the micro-scale of the cellular model. It may be either non-spatial, consisting, for example, of a model of economic and demographic change in the urban area as a whole, or spatial, as in the case of the standard spatial interaction based models operating on statistical units like census tracts. Only in the latter case, of course, can it possibly make up for the deficiencies of the cellular model described in the previous section. In either case, however, the macro-scale model constrains the cellular dynamics by providing target numbers for cells of each state, but in the case of the spatial model the targets may be regionally specific.

For example, a macro-scale model may generate a demand for a certain number of housing cells, with the actual locations to be determined by the cellular model; but if the macro-scale model is regionalized and so contains its own representation of spatial dynamics, then the model will allocate the total predicted demand among the regions, so that the cellular model then works with regional rather than global targets. 
The discussion in this section is based on experience with an integrated model consisting of the cellular model described above (Section 4) linked to a dynamic, regionalized spatial interaction based model of urban structure (Fig. 4). The interesting problems arise, of course, in linking the two levels. While sets of cells can be associated unambiguously with regions, the same is not necessarily true of the activities associated with the spatial units. State variables and parameters associated with cells can generally be aggregated in some appropriate way over all cells corresponding to a region and then assigned to that region in the macro-scale model. But if regional data is to be passed to the cellular model, it is frequently not possible to assign it exclusively to the set of cells corresponding to the region, as we will see below. Thus in general the relationship between an activity variable in the regional model and the regional expression of that variable in cell space is one-to-many. Furthermore, while in simple models there may be a one-to-one correspondence between the activity variables as defined in the regional model and the cell states defined in the CA model (e.g. population $\leftrightarrow$ housing), in general this is not the case: the population variable in the regional model may correspond to several CA cell states (e.g. population $\leftrightarrow[(1)$ single family housing, (2) low rise apartments, (3) high-rise apartments]), and vice versa.

A typical macro-scale model, specifically a regionalized, spatial interaction based model of urban structure dynamics, will re-allocate activity $\left(X_{i j}\right)$ - e.g. population, industry, offices, jobs, etc. from one region $(i)$ to another $(j)$ on the basis of the quantity of activity in each of the two regions $\left(X_{i}, X_{j}\right)$, the distance between them $\left(d_{i j}\right)$, and the distance weighted attraction of all competing regions $\left(V_{k}\right)$ :

$$
\begin{aligned}
& X_{i j}=f\left(X_{i}, X_{j}, d_{i j}, V_{i j}\right) \\
& \quad i, j, k=1, \ldots, R=\text { number of regions. }
\end{aligned}
$$
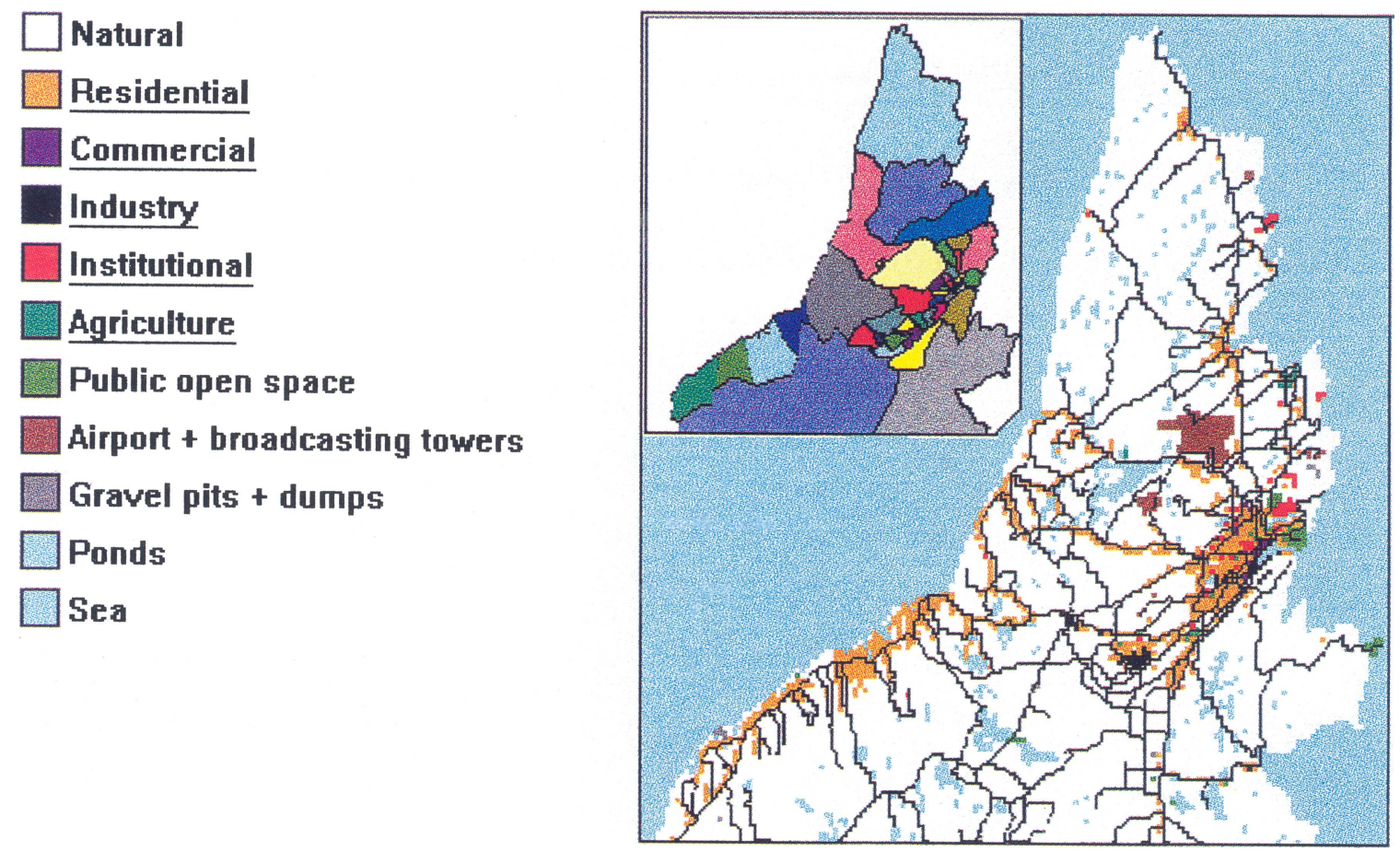

FIGURE 4 Simulation of St. John's (note the cellular structure of the land use map), with inset showing the 40 regions on which the macro-scale regional model operates. See Colour Plate III. 
But as Couclelis pointed out, one of the major shortcomings of such a model is that its space is purely relational; the model makes no use of data with absolute spatial coordinates - i.e. the sort of data that is stored in a GIS and is used to generate suitabilities for the CA model described above. Neither does the model incorporate possible effects of the micro-scale configurations generated by the cellular dynamics, nor does it take into account possible effects of crowding or land shortages. Thus when such a model is linked to a CA model there is an immediate potential for reformulating it so that it includes additional variables that reflect these cellular level phenomena:

$$
X_{i j}=g\left(X_{i}, X_{j}, d_{i j}, V_{i j}, w_{j i}, p_{j i}, s_{j i}\right)
$$

where $w_{j i}$ is the ratio of the density of activity in the destination region $j$ to that in the origin region $i, p_{j i}$ is the ratio of the means of the cellular potentials for the activity (as in Eq. (1)) in the two regions, and $s_{j i}$ is the ratio of the mean suitabilities of cells in the two regions for the activity.

What is gained by introducing these new variables that summarize information from the cellular model? Typical urban dynamics models as represented by Eq. (4) embody only positive feedback effects: in most formulations the movement of activity into a region is positively related to the existing level of activity, with no negative or inhibitory terms included. In particular, with the exception of the models due to Allen (1997), they typically do not include a density term. Yet density is probably one of the most important controls on urban dynamics, since as density increases, the associated costs, particularly the rising cost of land and the indirect costs of congestion, will eventually inhibit the continued growth of the most attractive regions.

Of course such effects can be included in the model, as Allen (1997) has demonstrated; but to do so in more than the most arbitrary way requires the sort of detailed spatial data which resides at the cellular level. For example, if density is obtained in the obvious way by dividing the level of activity by the area of the region, the measure will not be comparable from one region to another: in one region the entire area may be available to the activity, while in another only a small part may effectively be available, the rest being too steep, to wet, or occupied by an activity that cannot easily be dislodged. Thus meaningful densities must be defined at the cellular level; for example, the densities used to define $w_{j i}$ in Eq. (5) could be calculated as the level of activity in the region divided by the number of cells occupied by the activity, giving a measure of actual operating density that would be closely correlated with density associated costs.

Similarly, the terms $p_{j i}$ and $s_{j i}$ represent the comparative attractiveness of two regions in terms of local or cell scale characteristics within the regions. The relative mean potentials $\left(p_{j i}\right)$ of the cells in the two regions provide a summary measure of the relative attractiveness of the local cell neighbourhoods in the two regions, while the relative mean suitabilities $\left(s_{j i}\right)$ of the cells generalize the sitespecific qualities of Couclelis' absolute space to the regional level. Of course, all three of these variables summarizing cellular level properties can be specified in a variety of ways, and others could be defined as well.

The link from the macro-scale model to the cellular level serves primarily to provide the numbers of cells required for each activity. But in the case of the regionalized models, this is also the means by which the effects of long range interactions on the spatial restructuring of the city are transmitted to the cellular model, so that regional scale inhomogeneities are introduced into the cellular dynamics. There are a variety of ways in which the link can be specified, but all of them involve treating the regional activity levels generated by the macro-level model as demands $\left({ }^{t+1} X_{\mathrm{D} i}\right)$ rather than as actual new levels of activity, and then passing these demands to the cellular model for allocation in cell space and hence implicitly among regions.

A relatively loose connection between the two levels can be established by using the changes in demand in each region to re-scale the cellular 
potentials calculated in Eq. (1). Thus the potential values for cells in a region with a growing demand for an activity are rescaled upward relative to potentials for cells in regions with lower incremental demand, but the CA then runs as an unregionalized whole, constrained only by the total number of cells required for each sector. Thus in general the amount of activity implicitly allocated to each region by the cellular model will not be the same as the amount called for by the regional model, although regions with higher incremental demands $\left(\Delta^{t+1} X_{\mathrm{D} i}\right)$ will be relatively more attractive at the cellular level and thus will typically receive relatively more cells, and consequently more activity. The level of activity in a region is determined implicitly by the number of cells together with the density of activity per cell, so a final regional activity level $\left({ }^{t+1} X_{i}\right)$ as established in the CA can be calculated and returned to the regional model.

A tighter connection can be made by treating the regional demands for activities as regional targets at the cellular level. Regional activity demands $\left({ }^{t+1} X_{\mathrm{D} i}\right)$ are converted to regional demands for cells, and a regionalized CA attempts to allocate the cells within the specified regions. While in this approach the cellular model is regionalized with respect to target cell numbers, the calculation of cellular potentials (Eq. (1)) is made without regard to regional boundaries: the neighbourhood of a cell may include cells in another region. Thus it is possible, for example, for clusters of activity to develop which cross regional boundaries (Engelen et al., 1997a).

The regional targets approach is not so straightforward as it may seem, however, since it is not always possible to satisfy all of the demands for cells within a region. Thus there must be mechanisms for re-allocating some of the cell demand to other regions. These may take many forms, but typically they will be based on such factors as space available, existing regional demand for the activity $\left({ }^{t+1} X_{\mathrm{D} j}, j \neq i\right)$, density, and suitabilities. The intensity of the competition for cells in a region as measured by the ratio of cells demanded to cells available can be interpreted as a surrogate for land price. Since higher land prices lead to higher densities, densities are adjusted at each iteration of the cellular model to reflect the changing demand for land. Both the new densities and the derived final regional activity levels $\left({ }^{t+1} X_{i}\right)$ are returned to the macro-scale model.

We do not yet have sufficient experience with these linked models to know to what degree the performance of the component models is improved by the integration. Cellular models generally perform very well on their own, so it is to be expected that linking them with regionalized macro-scale models would not result in dramatic changes in their performance. The most noticeable improvements seem to be in sectors like commerce which are highly dependent on long range interactions and which have proved most difficult to model well with standalone CA. On the other hand, the spatial interaction based regional models may be greatly enhanced by integration with cellular models, primarily because the cellular models, taking into account a vast amount of detailed, high resolution spatial data concerning factors important to location, act to constrain the regional dynamics. The traditional spatial interaction based models, popular among planners in the 1950s and 1960s, have fallen out of favour, in part, perhaps because their results are not locationally specific; i.e. they have extremely poor resolution, and thus cannot easily be linked to specific urban phenomena. Potentially one of the major effects of linking these models to cellular models will be to revive their popularity.

More generally, there is no reason to limit integration to urban models. Cities are inherently complex entities, with socio-economic phenomena unfolding in an environment that is both constructed and natural. In the CA discussed so far, the built environment is modelled only in a general way (as land use), and the natural environment is treated as a set of boundary conditions, represented in the land cover features and the suitabilities. But the natural environment has its own dynamics, and in an urban area these dynamics are intimately linked to the socio-economic and land use dynamics of the constructed city. For example, 
a regional hydrological model may represent the dynamics of infiltration, runoff, stream discharge, and flooding as functions of precipitation and land cover. But the balance of infiltration and runoff is largely determined by land cover or land use, so as urbanization proceeds and land use changes, infiltration and runoff will be altered; in consequence groundwater supplies will be affected and so will the frequency and extent of flooding. But the latter effects may in turn feed back on the evolution of the urban area, for example by discouraging development of the areas that are newly at risk of flooding, or by instigating a planning intervention establishing a natural reserve dedicated to recharge of the local aquifer. Thus it is natural to link cellular urban models to models of natural processes (Uljee et al., 1996; White and Engelen, 1997a).

In spite of the specific difficulties that are always encountered, one of the great strengths of CA based models is the relative ease with which they can be linked to other models. Because process oriented models of many phenomena are inherently spatial and operate at local scales, as do CA, there is frequently a basic commensurability which facilitates integration. In this situation the output of one model may be passed to the other where it modifies a boundary condition; for example, in a case where forested land was being converted to commercial use, the output of an urban land use model would modify the local infiltration rate parameters of a hydrological model, which would thus express the effect of the land use change.

Even when the models are not commensurable, as is the case with regional models, or with non-spatial economic models (e.g. input-output models; see Engelen et al., 1997b; White and Engelen, 1997a), it is still possible, as we have seen, to establish strong links between the models. The reason lies in the rule-based nature of CA. Cellular models differ from each other primarily in terms of their transition rules, which can take on virtually any form, including hierarchical rule sets, in which one rule may over-ride another. This flexibility permits models to be linked through their rule sets, where the output of one model modifies the transition rules of the other. As the need for integrated modelling of human-natural systems becomes more obvious, it seems likely that the flexibility of CA will be seen as one of their most important characteristics.

\section{CONCLUSIONS}

Cellular automata constitute a powerful tool for understanding cities at more than one level. At the most mundane level, their high spatial resolution and micro-scale process modelling permit a high degree of verisimilitude. Such highly specific models, linked to the GIS that currently serve as the primary support for urban spatial planning, would radically extend the capabilities of the GIS by adding a temporal, predictive dimension to their high-resolution spatial database, thus enabling planners to experiment with possible urban futures.

But there is a deeper significance to the realism and practicality of CA based models. The history of urban modelling is plagued by the legacy of oversimplification. Starting with the standard land use models, which assumed a monocentric city lying on an isotropic plain, and continuing with the macro-scale urban dynamics models discussed above, which are defined on highly aggregated regions and thus lack spatial resolution, the results have been too general or too unrealistic to be of practical use.

More importantly, these traditional approaches have furnished relatively few fundamental insights into the nature of urban systems, primarily, it would seem, because they are too divorced from the nature of cities. As self-organizing structures, cities are inherently complex objects. Even the "simple" properties of cities cannot be understood if the underlying complexity is ignored. For example, the monocentric structure exhibited by many cities cannot be explained by a model that assumes monocentricity; nor can the fractal nature of cities be discerned in the absence of a representation of the underlying structural complexity. Thus a deeper theoretical understanding can only be achieved 
through methods that bring together the general and the specific - methods that capture and work with the detail and complexity that characterize real cities.

Extensive theoretical work on the principles of self-organization by Langton (1990; 1992), Kauffman (1989; 1990; 1993), and others (see, e.g. Forrest, 1991), using CA and related techniques, has begun to provide deep insights into the behaviour of whole classes of complex systems. There are, for example, strong indications that evolved complex systems will typically have fractal characteristics. In this light, then, it is not surprising that cities have a fractal structure, or that cellular models of cities generate such a structure (and instructive that traditional urban models do not). The point is that cellular techniques dramatically shrink the distance between highly specific models of actual cities and models formulated to investigate fundamental theoretical issues. They may be expected to lead to richer and more useful applied models co-evolving with a deeper understanding of urban systems.

\section{References}

Allen, P. (1983). "Self-organization and evolution in urban systems". Crosby, R. (ed.): Cities and Regions as Nonlinear Decision Systems. Westview Press.

Allen, P. (1997). Cities and Regions as Self-Organizing Systems: Models of Complexity. Gordon and Breach.

Bak, P., Chen, K. and Creutz, M. (1989). "Self-organized criticality in the 'game of life"'. Nature 342, 780-782.

Batty, M. and Longley, P. (1994). Fractal Cities. Academic Press.

Batty, M. and Xie, Y. (1994). "From Cells to Cities". Environment and Planning B 21, 31-48.

Batty, M. and Xie, Y. (1996). "Possible cellular automata". Besussi, E. and Cecchini, A. (eds.): Artificial Worlds and Urban Studies. Venice: DAEST, pp. 191-220.

Cecchini, A. (1996). "Approaching generalized urban automata with help! on line (AUGH)". Besussi, E. and Cecchini, A. (eds.): Artificial Worlds and Urban Studies. Venice: DAEST, pp. 231-248.

Cecchini, A. and Viola, F. (1990). "Eine stadtbausimulation". Wissenschaftliche Zeitschrift der Hochschule für Architektur und Bauwesen 36(4), 159-162.

Cecchini, A. and Viola, F. (1992). "Ficties (Fictitious Cities): A simulation for the creation of cities". Paper presented at the International Seminar on Cellular Automata for Regional Analysis, DAEST, Universitario di Architettura, Venice.

Couclelis, H. (1985). "Cellular worlds: a framework for modeling micro-macro dynamics". Environment and Planning A 17, 585-596.
Couclelis, H. (1988). "Of mice and men: what rodent populations can teach us about complex spatial dynamics”. Environment and Planning A 20, 99-109.

Couclelis, H. (1989). "Macrostructure and microbehavior in a metropolitan area". Environment and Planning B: Planning and Design 16, 141-154.

Couclelis, H. (1996). "From cellular automata to urban models: new principles for model development and implementation". Besussi, E. and Cecchini, A. (eds.): Artificial Worlds and Urban Studies. Venice: DAEST, pp. 165-190.

DiGregorio, S., Festa, D., Gattuso, D., Rongo, R., Spataro, W., Spezzano, G. and Vitetta, A. (1996). "Cellular automata for freeway traffic simulation". Besussi, E. and Cecchini, A. (eds.): Artificial Worlds and Urban Studies. Venice: DAEST, pp. 365-392.

Engelen, G., White, R. and Uljee, I. (1997a). "Integrating constrained cellular automata models, GIS and decision support tools for urban and regional planning and policy making". Timmermans, H. (ed.): Decision Support Systems in Urban Planning. London: E \& FN Spon. pp. 125-155.

Engelen, G., White, R. and Uljee, I. (1997b). Vulnerability Assessment of Low-Lying Coastal Areas and Small Islands to Climate Change and Sea Level Rise, Phase 2: Case Study St. Lucia. Report to UNEP CAR/RCU. Maastricht: Research Institute for Knowledge Systems.

Forrest, S. (ed.). (1991). Emergent Computation. MIT/North Holland.

Frankhauser, P. (1991). "Aspects fractals des structures urbaines". L'Espace Geographique, 1, 45-69.

Frankhauser, P. (1994). La Fractilité des structures urbaines. Paris: Economica.

Kauffman, S. (1989). "Adaption on rugged fitness landscapes". Stein, P. (ed.): Lectures in the Sciences of Complexity. Addison-Wesley, pp. 527-618.

Kauffman, S. (1990). "Requirements for evolvability in complex systems: orderly dynamics and frozen components". Physica D 42, 135-152.

Kauffman, S. (1993). The Origins of Order. Oxford University Press.

Langton, C. (1990). "Computation at the edge of chaos: phase transitions and emergent computation". Physica D 42, 12-37.

Langton, C. (1992). "Life at the edge of chaos". Langton, C. et al. (eds.): Artificial Life II: Proceedings of an Interdisciplinary Workshop on the Synthesis and Simulation of Living Systems, Santa Fe Institute Studies in the Science of Complexity, V. 10. Redwood City: Addison-Wesley. pp. 41-92.

Makse, H., Shlomo, H. and Stanley, E. (1995). "Modelling urban growth patterns". Nature 377, 608-612.

Mandelbrot, B. (1983). The Fractal Geometry of Nature. New York: W.H. Freeman.

Monserud, R.A. and Leemans, R. (1992). "Comparing glogal vegetation maps with the kappa statistic". Ecological Modelling 62, 275-293.

Nagel, K., Rasmussen, S. and Barrett, C. (1997). "Network traffic as a self-organized critical phenomenon". Schweitzer, F. and Haken, H. (eds.): Self-Organization of Complex Structures: from Individual to Collective Dynamics. Gordon and Breach, pp. 579-592.

Nicolis, G. (1989). "Physics of far-from-equilibrium systems and self-organization". Davies, P. (ed.): The New Physics. Cambridge University Press, pp. 316-347.

Portugali, J. and Benenson, I. (1995). "Artificial planning experience by means of a heuristic cell-space model: simulating international migration in the urban process". Environment and Planning A 27, 1647-1665. 
Portugali, J. and Benenson, I. (1997). "Human agents between local and global forces in a self-organizing city". Schweitzer, F. and Haken, H. (eds.): Self-Organization of Complex Structures: from Individual to Collective Dynamics. Gordon and Breach, pp. 537-545.

Portugali, J., Benenson, I. and Omer, I. (1994). "Socio-spatial residential dynamics: stability and instability within a selforganizing city". Geographical Analysis 26, 321-340.

Power, C. (1998). Hierarchical Fuzzy Pattern Matching for the Regional Comparison of Land Use Maps. Memorial University of Newfoundland: Unpublished Thesis.

Takeyama, M. (1996). "Geocellular: a general platform for dynamic spatial simulation". Besussi, E. and Cecchini, A. (eds.): Artificial Worlds and Urban Studies. Venice: DAEST, pp. 347-364.

Tobler, W. (1979). "Cellular geography”. Gale, S. and Olsson, G. (eds.), Philosophy in Geography. pp. 379-386.

Uljee, I., Engelen, G. and White, R. (1996). Ramco Demo Guide. Workdocument CZM0C 96.08. The Hague: Coastal Zone Management Centre, National Institute for Coastal and Marine Management.

White, R. (1977). "Dynamic central place theory: results of a simulation approach". Geographical Analysis 9, 227-243.

White, R. (1978). "The simulation of central place dynamics: two sector systems and the rank-size distribution". Geographical Analysis 10, 201-208.

White, R. and Engelen, G. (1993a). "Cellular dynamics and GIS: modelling spatial complexity". Geographical Systems 1, 237-253.
White, R. and Engelen, G. (1993b). "Fractal urban land use patterns: a cellular automata approach". Environment and Planning A 25, 1175-1199.

White, R. and Engelen, G. (1994). "Urban systems dynamics and cellular automata: fractal structures between order and chaos". Chaos, Solitons, and Fractals 4, 563-583.

White, R. and Engelen, G. (1997a). "Cellular automata as the basis of integrated dynamic regional modelling". Environment and Planning B 24, 235-246.

White, R. and Engelen, G. (1997b). "Multi-scale spatial modelling of self-organizing urban systems". Schweitzer, F. and Haken, H. (eds.): Self-Organization of Complex Structures: from Individual to Collective Dynamics. Gordon and Breach, pp. 519-535.

White, R., Engelen, G. and Uljee, I. (1997). "The use of constrained cellular automata for high-resolution modelling of urban land-use dynamics". Environment and Planning B 24, $323-343$.

Wilson, A. (1974). Urban and Regional Models in Geography and Planning. London: John Wiley and Sons.

Wilson, A. (1976). "Retailers' profits and consumers' welfare in a spatial interaction shopping model". Masser, I. (ed.): London Papers in Regional Science 6: Theory and Practice in Regional Science. London: Pion, pp. 42-59.

Wolfram, S. (1984). "Universality and complexity in cellular automata". Physica D 10, 1-35.

Xie, Y. (1996). "A generalized model for cellular urban dynamics". Geographical Analysis 28, 350-373. 


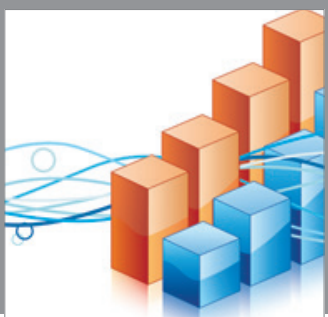

Advances in

Operations Research

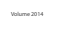

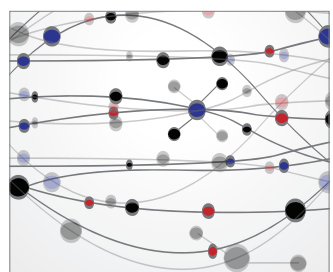

\section{The Scientific} World Journal
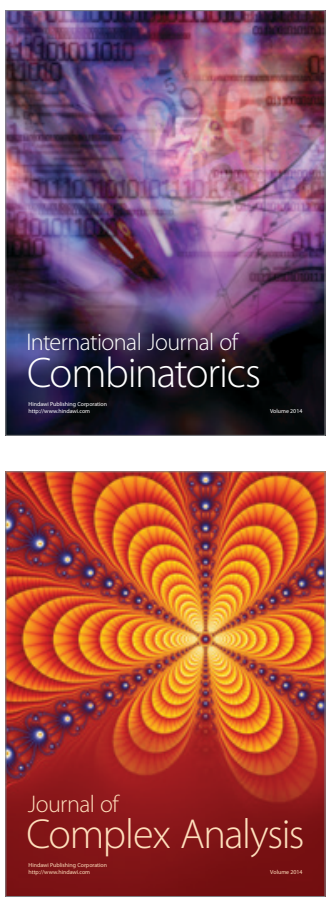

International Journal of

Mathematics and

Mathematical

Sciences
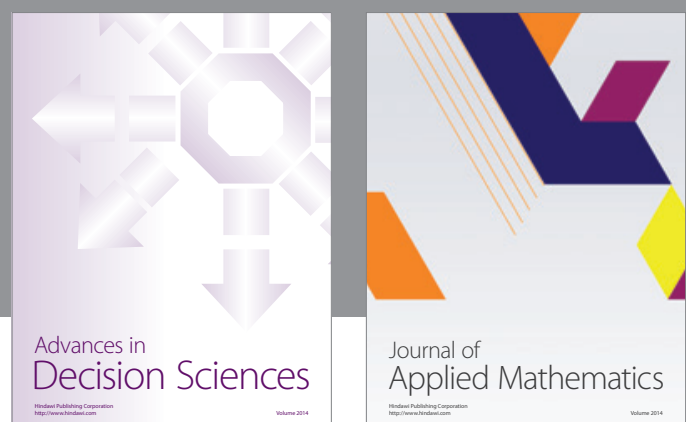

Journal of

Applied Mathematics
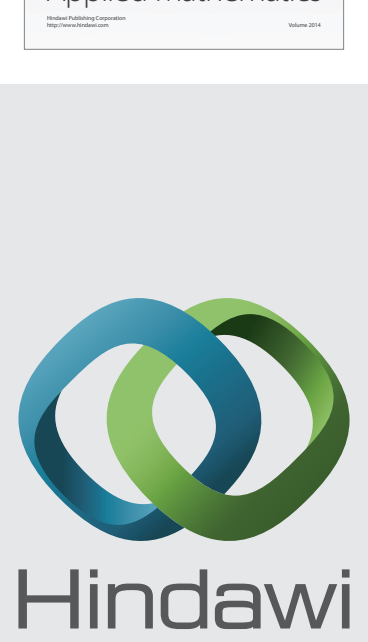

Submit your manuscripts at http://www.hindawi.com
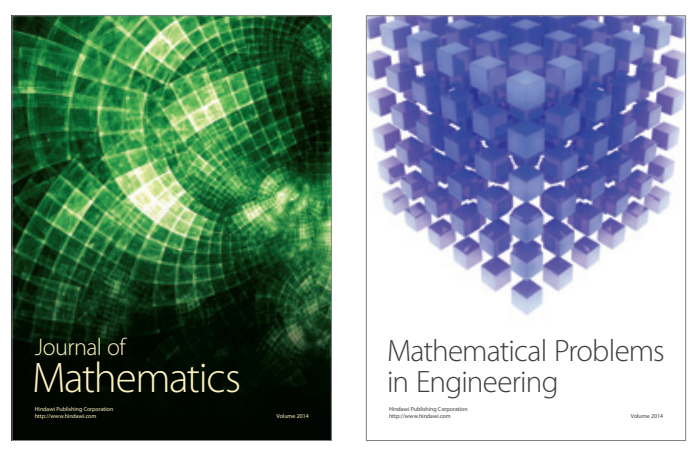

Mathematical Problems in Engineering
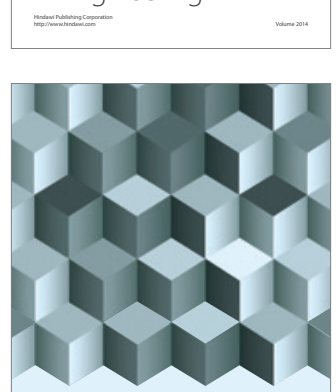

Journal of

Function Spaces
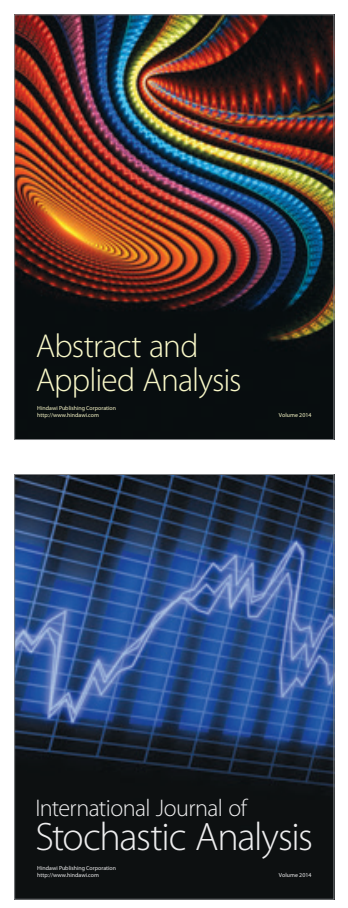

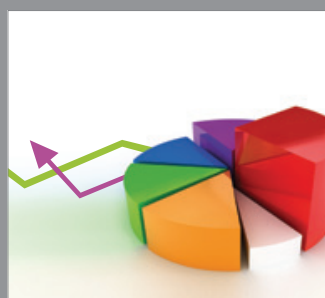

ournal of

Probability and Statistics

Promensencen
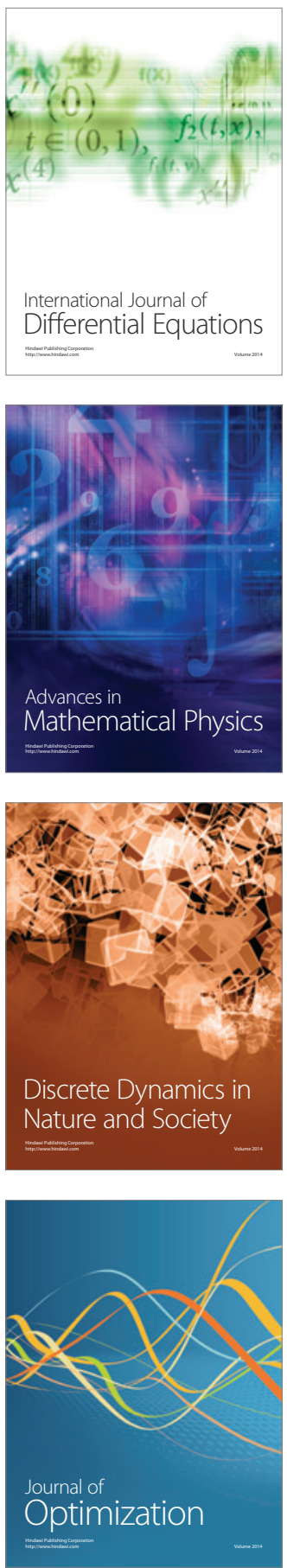